TRANSACTIONS OF THE

AMERICAN MATHEMATICAL SOCIETY

Volume 360, Number 11, November 2008, Pages 5789-5803

S 0002-9947(08)04441-3

Article electronically published on May 28, 2008

\title{
A GEOMETRIC DESCRIPTION OF $m$-CLUSTER CATEGORIES
}

\author{
KARIN BAUR AND BETHANY R. MARSH
}

\begin{abstract}
We show that the $m$-cluster category of type $A_{n-1}$ is equivalent to a certain geometrically defined category of diagonals of a regular $n m+2$-gon. This generalises a result of Caldero, Chapoton and Schiffler for $m=1$. The approach uses the theory of translation quivers and their corresponding mesh categories. We also introduce the notion of the $m$-th power of a translation quiver and show how it can be used to realise the $m$-cluster category in terms of the cluster category.
\end{abstract}

\section{INTRODUCTION}

Let $n, m \in \mathbb{N}$ and let $\Pi$ be a regular $n m+2$-sided polygon. We show that a category $\mathcal{C}_{A_{n-1}}^{m}$ of diagonals can be associated to $\Pi$ in a natural way. The objects of $\mathcal{C}_{A_{n-1}}^{m}$ are the diagonals in $\Pi$ which divide $\Pi$ into two polygons whose numbers of sides are congruent to 2 modulo $m$, as considered in [PS]. A quiver $\Gamma_{A_{n-1}}^{m}$ can be defined on the set of such diagonals, with arrows given by a simple geometrical rule. It is shown that this quiver is a stable translation quiver in the sense of Riedtmann [Rie] with translation $\tau$ given by a certain rotation of the polygon. For a field $k$, the category $\mathcal{C}_{A_{n-1}}^{m}$ is defined as the mesh category associated to $\left(\Gamma_{A_{n-1}}^{m}, \tau\right)$.

Let $Q$ be a Dynkin quiver of type $A_{n-1}$, and let $D^{b}(k Q)$ denote the bounded derived category of finite dimensional $k Q$-modules. Let $\tau$ denote the Auslander-Reiten translate of $D^{b}(k Q)$, and let $S$ denote the shift. These are both autoequivalences of $D^{b}(k Q)$. Our main result is that $\mathcal{C}_{A_{n-1}}^{m}$ is equivalent to the quotient of $D^{b}(k Q)$ by the autoequivalence $\tau^{-1} S^{m}$. We thus obtain a geometric description of this category in terms of $\Pi$.

The $m$-cluster category $D^{b}(k Q) / \tau^{-1} S^{m}$ associated to $k Q$ was introduced in [Kel] and has also been studied by Thomas [Tho], Wralsen [Wra] and Zhu [Zhu]. It is a generalisation of the cluster category defined in [CCS1] (for type $A$ ) and [BMRRT] (the general hereditary case). Keller has shown that it is a Calabi-Yau category of

Received by the editors July 26, 2006.

2000 Mathematics Subject Classification. Primary 16G20, 16G70, 18E30; Secondary 05E15, $17 \mathrm{~B} 37$.

Key words and phrases. Cluster category, $m$-cluster category, polygon dissection, $m$-divisible, cluster algebra, simplicial complex, mesh category, diagonal, Auslander-Reiten quiver, derived category, triangulated category.

This research was supported by Engineering and Physical Sciences Research Council grant GR/S35387/01. 
dimension $m+1$ [Kel]. We remark that such Calabi-Yau categories have also been studied in $[\mathrm{KR}]$.

Our definition is motivated by and is a generalisation of the construction of the cluster category in type $A$ given in [CCS1], where a category of diagonals of a polygon is introduced. The authors show that this category is equivalent to the cluster category associated to $k Q$. This can be regarded as the case $m=1$ here. The aim of the current paper is to generalise the construction of [CCS1] to the diagonals arising in the $m$-divisible polygon dissections considered in [PS]. Note that Tzanaki [Tza] has also studied such diagonals. We also remark that a connection between the $m$-cluster category associated to $k Q$ and the diagonals considered here was given in [Tho].

We further show that if $(\Gamma, \tau)$ is any stable translation quiver, then the quiver $\Gamma^{m}$ with the same vertices but with arrows given by sectional paths in $\Gamma$ of length $m$ is again a stable translation quiver with translation given by $\tau^{m}$. If $(\Gamma, \tau)$ is taken to be the Auslander-Reiten quiver of the cluster category of a Dynkin quiver of type $A_{n m-1}$, we show that $\Gamma^{m}$ contains $\Gamma_{A_{n-1}}^{m}$ as a connected component. It follows that the $m$-cluster category is a full subcategory of the additive category generated by the mesh category of $\Gamma^{m}$.

Since $\Gamma$ is known to have a geometric construction [CCS1], our definition provides a geometric construction for the additive category generated by the mesh category of any connected component of $\Gamma^{m}$. We give an example to show that this provides a geometric construction for quotients of $D^{b}(k Q)$ other than the $m$-cluster category.

\section{Notation AND DEFinitions}

In [Tza], E. Tzanaki studied an abstract simplicial complex obtained by dividing a polygon into smaller polygons.

We recall the definition of an abstract simplicial complex. Let $X$ be a finite set and $\triangle \subseteq \mathcal{P}(X)$ a collection of subsets. Assume that $\triangle$ is closed under taking subsets (i.e. if $A \in \triangle$ and $B \subseteq A$, then $B \in \triangle$ ). Then $\triangle$ is an abstract simplicial complex on the ground set $X$. The vertices of $S$ are the single element subsets of $\triangle$ (i.e. $\{A\} \in \triangle$ ). The faces are the elements of $\triangle$, and the facets are the maximal among those (i.e. the $A \in \triangle$ such that if $A \subseteq B$ and $B \in \triangle$, then $A=B$ ). The dimension of a face $A$ is equal to $|A|-1$ (where $|A|$ is the cardinality of $A$ ). The complex is said to be pure of dimension $d$ if all its facets have dimension $d$.

Let $\Pi$ be an $n m+2$-gon, $m, n \in \mathbb{N}$, with vertices numbered clockwise from 1 to $n m+2$. We regard all operations on vertices of $\Pi$ modulo $n m+2$. A diagonal $D$ is denoted by the pair $(i, j)$ (or simply by the pair $i j$ if $1 \leq i, j \leq 9$ ). Thus $(i, j)$ is the same as $(j, i)$. We call a diagonal $D$ in $\Pi$ an $m$-diagonal if $D$ divides $\Pi$ into an $(m j+2)$-gon and an $(m(n-j)+2)$-gon where $j=1, \ldots,\left\lceil\frac{n-1}{2}\right\rceil$. Then Tzanaki defines the abstract simplicial complex $\triangle=\triangle_{A_{n-1}}^{m}$ on the $m$-diagonals of $\Pi$ as follows.

The vertices of $\triangle$ are the $m$-diagonals. The faces of $\triangle_{A_{n-1}}^{m}$ are the sets of $m$ diagonals which pairwise do not cross. They are called $m$-divisible dissections (of $\Pi$ ). Then the facets are the maximal collections of such $m$-diagonals. Each facet contains exactly $n-1$ elements, so the complex $\triangle_{A_{n-1}}^{m}$ is pure of dimension $n-2$.

The case $m=1$ is the complex whose facets are triangulations of an $n+2$-gon. 


\section{A stable translation Quiver of Diagonals}

To $\triangle=\triangle_{A_{n-1}}^{m}$ we associate a category along the lines of [CCS1]. As a first step, we associate to the simplicial complex a quiver, called $\Gamma_{A_{n-1}}^{m}$. The vertices of the quiver are the $m$-diagonals in the defining polygon $\Pi$, i.e. the vertices of $\triangle_{A_{n-1}}^{m}$.

The arrows of $\Gamma_{A_{n-1}}^{m}$ are obtained in the following way:

Let $D, D^{\prime}$ be $m$-diagonals with a common vertex $i$ of $\Pi$. Let $j$ and $j^{\prime}$ be the other endpoints of $D$, respectively $D^{\prime}$. The points $i, j, j^{\prime}$ divide the boundary of the polygon $\Pi$ into three arcs, linking $i$ to $j, j$ to $j^{\prime}$ and $j^{\prime}$ to $i$. (We usually refer to a part of the boundary connecting one vertex to another as an arc.) If $D, D^{\prime}$ and the arc from $j$ to $j^{\prime}$ form an $m+2$-gon in $\Pi$ and if, furthermore, $D$ can be rotated clockwise to $D^{\prime}$ about the common endpoint $i$, we draw an arrow from $D$ to $D^{\prime}$ in $\Gamma_{A_{n-1}}^{m}$. (By this we mean that $D$ can be rotated clockwise to the line through $D^{\prime}$.) Note that if $D, D^{\prime}$ are vertices of the quiver $\Gamma_{A_{n-1}}^{m}$, then there is at most one arrow between them.

Examples 2.4 and 2.5 below illustrate this construction.

We then define an automorphism $\tau_{m}$ of the quiver: let $\tau_{m}: \Gamma_{A_{n-1}}^{m} \rightarrow \Gamma_{A_{n-1}}^{m}$ be the map given by $D \mapsto D^{\prime}$ if $D^{\prime}$ is obtained from $D$ by an anticlockwise rotation through $\frac{2 m \pi}{n m+2}$ about the centre of the polygon. Clearly, $\tau_{m}$ is a bijective map and a morphism of quivers.

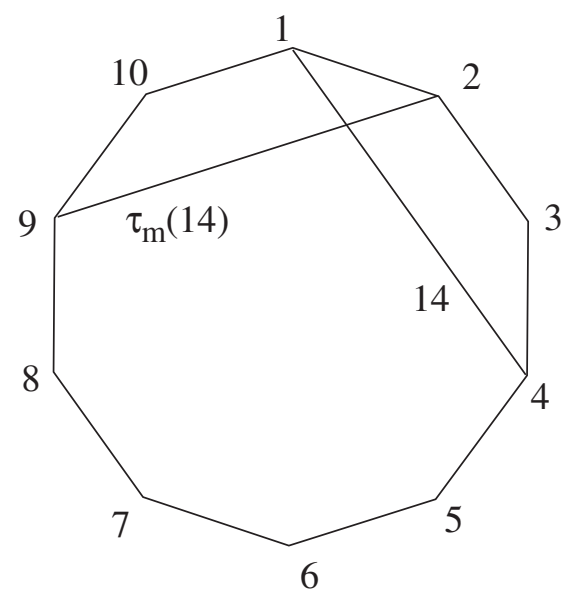

FiguRE 1. The translation $\tau_{m}, \tau_{m}(14)=92$, where $n=4, m=2$

Definition 2.1. (1) A translation quiver is a pair $(\Gamma, \tau)$ where $\Gamma$ is a locally finite quiver and $\tau: \Gamma_{0}^{\prime} \rightarrow \Gamma_{0}$ is an injective map defined on a subset $\Gamma_{0}^{\prime}$ of the vertices of $\Gamma$ such that for any $X \in \Gamma_{0}, Y \in \Gamma_{0}^{\prime}$, the number of arrows from $X$ to $Y$ is the same as the number of arrows from $\tau(Y)$ to $X$. The vertices in $\Gamma_{0} \backslash \Gamma_{0}^{\prime}$ are called projective. If $\Gamma_{0}^{\prime}=\Gamma_{0}$ and $\tau$ is bijective, $(\Gamma, \tau)$ is called a stable translation quiver.

(2) A stable translation quiver is said to be connected if it is not a disjoint union of two non-empty stable subquivers.

Proposition 2.2. The pair $\left(\Gamma=\Gamma_{A_{n-1}}^{m}, \tau_{m}\right)$ is a stable translation quiver. 
Proof. By definition, $\tau_{m}$ is a bijective map from $\Gamma$ to $\Gamma$, and $\Gamma$ is a finite quiver. We have to check that the number of arrows from $D$ to $D^{\prime}$ in $\Gamma$ is the same as the number of arrows from $\tau_{m} D^{\prime}$ to $D$. Since there is at most one arrow from one vertex to another, we only have to see that there is an arrow $D \rightarrow D^{\prime}$ if and only if there is an arrow $\tau_{m} D^{\prime} \rightarrow D$.

Assume that there is an arrow $D \rightarrow D^{\prime}$, and let $i$ be the common vertex of $D$ and $D^{\prime}$ in the polygon, $D=(i, j), D^{\prime}=(i, j+m)$. Then $\tau_{m} D^{\prime}=(i-m, j)$. In particular, $j$ is the common vertex of $D$ and $\tau_{m} D^{\prime}$. Furthermore, we obtain $D$ from $\tau_{m} D^{\prime}$ by a clockwise rotation about $j$, and these two $m$-diagonals form an $m+2$-gon together with an arc from $i-m$ to $i$; hence there is an arrow $\tau_{m} D^{\prime} \rightarrow D$. See Figure 2.

The converse follows with the same reasoning.

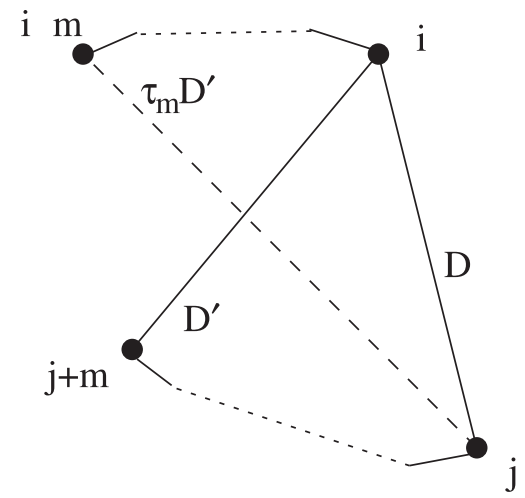

Figure 2. $D \rightarrow D^{\prime} \Longleftrightarrow \tau_{m} D^{\prime} \rightarrow D$

Proposition 2.3. $\left(\Gamma, \tau_{m}\right)$ is a connected stable translation quiver.

Proof. Note that every vertex of $\Pi$ is incident with some element of any given $\tau_{m}$-orbit of $m$-diagonals: any $m$-diagonal is of the form $(i, i+k m+1)$ and

$$
\begin{aligned}
\tau_{m}^{k-n}(i, i+k m+1) & =(i+(n-k) m, i+n m+1) \\
& =(i+(n-k) m, i-1) .
\end{aligned}
$$

Assume that $\Gamma$ is the disjoint union of two non-empty stable subquivers. So there exist $m$-diagonals $D=(i, j)$ and $D^{\prime}=\left(i^{\prime}, j^{\prime}\right)$ that cannot be connected by any path in $\Gamma$. After rotating $D^{\prime}$ using $\tau_{m}$ we can assume that $i=i^{\prime}$. By assumption, $j^{\prime} \neq j+r m$ for any $r$. Without loss of generality, $j<j^{\prime}$. The diagonal $D$ can be rotated clockwise about $i$ to another $m$-diagonal $D^{\prime \prime}=\left(i, j^{\prime \prime}\right)$ such that $j^{\prime}=j^{\prime \prime}+s$ with $0<s<m$. Since $D^{\prime \prime}$ is an $m$-diagonal, the arc from $i$ to $j^{\prime \prime}$, not including $j^{\prime}$, together with $D^{\prime \prime}$, bounds a $(u m+2)$-gon for some $u$. But then the arc from $i$ to $j^{\prime}$, including $j^{\prime \prime}$, together with the diagonal $D^{\prime}$, bound a $(u m+2+s)$-gon where $u m+2<u m+2+s<(u+1) m+2$. Hence $D^{\prime}$ cannot be an $m$-diagonal.

In the examples below we draw the quiver associated to the complex $\triangle_{A_{n-1}}^{m}$ in the standard way of Auslander-Reiten theory: the vertices and arrows are arranged so that the translation $\tau_{m}$ is a shift to the left. We indicate it by dotted lines. 
Example 2.4. Let $n=4, m=1$, i.e. $\Pi$ is a 6 -gon. The rotation group given by rotation about the centre of $\Pi$ through $k \times \frac{\pi}{3}$ degrees $(k=0, \ldots, 5)$ acts on the facets of $\triangle_{A_{3}}^{1}$. There are four orbits, $\mathcal{O}_{\{13,14,15\}}$ of size $6, \mathcal{O}_{\{13,14,46\}}$ and $\mathcal{O}_{\{13,36,46\}}$ of size 3 and $\mathcal{O}_{\{13,15,35\}}$ with two elements, making a total of 14 elements.

The vertices of the quiver $\Gamma_{A_{3}}^{1}$ are the nine 1-diagonals $\{13,14,15,24,25,26,35$, $36,46\}$, and we draw the quiver as follows:

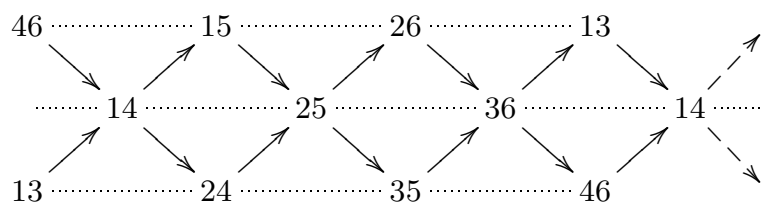

Example 2.5. Let $m=2$ and $n=4$, i.e. $\Pi$ is a 10 -gon. The rotation group is given by the rotations about the centre of $\Pi$ through $k \times \frac{2 \pi}{5}$ degrees $(k=0, \ldots, 9)$ and acts on the facets of $\triangle_{A_{3}}^{2}$. The orbits are $\mathcal{O}_{\{14,16,18\}}, \mathcal{O}_{\{14,18,47\}}, \mathcal{O}_{\{18,38,47\}}$ and $\mathcal{O}_{\{47,38,39\}}$ of size 10 , and $\mathcal{O}_{\{14,16,69\}}, \mathcal{O}_{\{14,49,69\}}$ and $\mathcal{O}_{\{29,38,47\}}$ of size 5 , making a total of 55 elements. The vertices of $\Gamma_{A_{3}}^{2}$ are the fifteen 2-diagonals $\{14,16,18,25,27,29,36,38,(3,10), 47,49,58,(5,10), 69,(7,10)\}$ and the quiver is

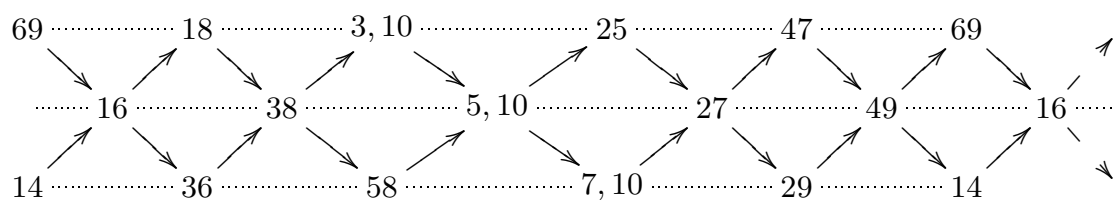

\section{3. $m$-CLUSTER CATEGORIES}

Let $G$ be a simply laced Dynkin diagram with vertices $I$. Let $Q$ be a quiver with underlying graph $G$, and let $k$ be an algebraically closed field. Let $k Q$ be the corresponding path algebra. Let $D^{b}(k Q)$ denote the bounded derived category of finitely generated $k Q$-modules, with shift denoted by $S$ and Auslander-Reiten translate given by $\tau$. It is known that $D^{b}(k Q)$ is triangulated, Krull-Schmidt and has almost-split triangles (see [Hap]). Let $\mathbb{Z} Q$ be the stable translation quiver associated to $Q$, with vertices $(n, i)$ for $n \in \mathbb{Z}$ and $i$ a vertex of $Q$. For every arrow $\alpha: i \rightarrow j$ in $Q$ there are arrows $(n, i) \rightarrow(n, j)$ and $(n, j) \rightarrow(n+1, i)$ in $\mathbb{Z} Q$, for all $n \in \mathbb{Z}$. Together with the translation $\tau$, taking $(n, i)$ to $(n-1, i), \mathbb{Z} Q$ is a stable translation quiver. We note that $\mathbb{Z} Q$ is independent of the orientation of $Q$ and can thus be denoted $\mathbb{Z} G$.

We recall the notion of the mesh category of a stable translation quiver with no multiple arrows (the mesh category is defined for a general translation quiver, but we shall not need that here). Recall that for a quiver $\Gamma, k\langle\Gamma\rangle$ denotes the path category on $\Gamma$, with morphisms given by arbitrary $k$-linear combinations of paths.

Definition 3.1. Let $(\Gamma, \tau)$ be a stable translation quiver with no multiple arrows. Let $Y$ be a vertex of $\Gamma$ and let $X_{1}, \ldots, X_{k}$ be all the vertices with arrows to $Y$, denoted $\alpha_{i}: X_{i} \rightarrow Y$. Let $\beta_{i}: \tau(Y) \rightarrow X_{i}$ be the corresponding arrows from $\tau(Y)$ to $X_{i}(i=1, \ldots, k)$. Then the mesh ending at $Y$ is defined to be the quiver consisting of the vertices $Y, \tau(Y), X_{1}, \ldots, X_{k}$ and the arrows $\alpha_{1}, \alpha_{2}, \ldots, \alpha_{k}$ and 
$\beta_{1}, \beta_{2}, \ldots, \beta_{k}$. The mesh relation at $Y$ is defined to be

$$
m_{Y}:=\sum_{i=1}^{k} \beta_{i} \alpha_{i} \in \operatorname{Hom}_{k\langle\Gamma\rangle}(\tau(Y), Y) .
$$

Let $J_{m}$ be the ideal in $k\langle\Gamma\rangle$ generated by the mesh relations $m_{Y}$ where $Y$ runs over all vertices of $\Gamma$.

Then the mesh category of $\Gamma$ is defined as the quotient $k\langle\Gamma\rangle / J_{m}$.

For an additive category $\varepsilon$, denote by ind $\varepsilon$ the full subcategory of indecomposable objects. Happel [Hap] has shown that ind $D^{b}(k Q)$ is equivalent to the mesh category of $\mathbb{Z} Q$, from which it follows that it is independent of the orientation of $Q$. Its Auslander-Reiten quiver is $\mathbb{Z} G$.

For $m \in \mathbb{N}$, we denote by $\mathcal{C}_{G}^{m}$ the $m$-cluster category associated to the Dynkin diagram $G$, so

$$
\mathcal{C}_{G}^{m}=\frac{D^{b}(k Q)}{F_{m}},
$$

where $Q$ is any orientation of $G$ and $F_{m}$ is the autoequivalence $\tau^{-1} \circ S^{m}$ of $D^{b}(k Q)$. This was introduced by Keller [Kel] and has been studied by Thomas [Tho], Wralsen [Wra] and Zhu [Zhu]. It is known that $\mathcal{C}_{G}^{m}$ is triangulated [Kel], Krull-Schmidt and has almost split triangles [BMRRT, 1.2,1.3]. Let $\varphi_{m}$ denote the automorphism of $\mathbb{Z} G$ induced by the autoequivalence $F_{m}$. The Auslander-Reiten quiver of $\mathcal{C}_{G}^{m}$ is the quotient $\mathbb{Z} G / \varphi_{m}$, and ind $\mathcal{C}_{G}^{m}$ is equivalent to the mesh category of $\mathbb{Z} G / \varphi_{m}$.

\section{Coloured Almost positive roots}

Our main aim in the next two sections is to show that, if $G$ is of type $A_{n-1}$, then ind $\mathcal{C}_{G}^{m}$ is equivalent to the mesh category $\mathcal{D}_{A_{n-1}}^{m}$ of the stable translation quiver $\Gamma_{A_{n-1}}^{m}$ defined in the previous section. From the previous section we can see that it is enough to show that, as translation quivers, $\mathbb{Z} G / \varphi_{m}$ is isomorphic to $\Gamma_{A_{n-1}}^{m}$. In this section, we recall the discussion of $m$-diagonals and $m$-coloured almost positive roots in Fomin-Reading [FR].

4.1. $m$-coloured almost positive roots and $m$-diagonals. For $\Phi$ a root system, with positive roots $\Phi^{+}$and simple roots $\alpha_{1}, \alpha_{2}, \ldots, \alpha_{n}$, let $\Phi_{>-1}^{m}$ denote the set of $m$-coloured almost positive roots (see [FR]). An element of $\Phi_{\geq-1}^{m}$ is either a $m$-coloured positive root $\alpha^{k}$ where $\alpha \in \Phi^{+}$and $k \in\{1,2, \ldots, m\}$ or a negative simple root $-\alpha_{i}$ for some $i$ which we regard as having colour 1 for convenience (it is thus also denoted $-\alpha_{i}^{1}$ ). Fomin-Reading [FR] show that there is a one-to-one correspondence between $m$-diagonals of the regular $n m+2$-gon $\Pi$ and $\Phi_{\geq-1}^{m}$ when $\Phi$ is of type $A_{n-1}$. We now recall this correspondence.

Recall that $R_{m}$ denotes the anticlockwise rotation of $\Pi$ taking vertex $i$ to vertex $i-1$ for $i \geq 2$, and vertex 1 to vertex $n m+2$. For $1 \leq i \leq \frac{n}{2}$, the negative simple root $-\alpha_{2 i-1}$ corresponds to the diagonal $((i-1) m+1,(n-i) m+2)$. For $1 \leq i \leq \frac{n-1}{2}$, the negative simple root $-\alpha_{2 i}$ corresponds to the diagonal $(i m+1,(n-i) m+2)$. Together, these diagonals form what is known as the $m$-snake; cf. Figure 3. For $1 \leq i \leq j \leq n$, there are exactly $m m$-diagonals intersecting the diagonals labelled $-\alpha_{i},-\alpha_{i+1}, \ldots,-\alpha_{j}$ and no other diagonals labelled with negative simple roots. These diagonals are of the form $D, R_{m}^{1}(D), \ldots, R_{m}^{m-1}(D)$ for some diagonal $D$, and $\alpha^{k}$ corresponds to $R_{m}^{k-1}(D)$ for $k=1,2, \ldots, m$, where $\alpha$ denotes the positive 
root $\alpha_{i}+\cdots+\alpha_{j}$. For an $m$-coloured almost positive root $\beta^{k}$, we denote the corresponding diagonal by $D\left(\beta^{k}\right)$.

It is clear that, for $1 \leq i \leq \frac{n}{2}$, the coloured root $\alpha_{2 i-1}^{1}$ corresponds to the diagonal $(i m+1,(n+1-i) m+2)$. Also, the diagonals $D\left(-\alpha_{i}\right)$, for $i$ even, together with $D\left(\alpha_{j}^{1}\right)$, for $j$ odd, form a 'zig-zag' dissection of $\Pi$ which we call the opposite m-snake; cf. Figure 3.
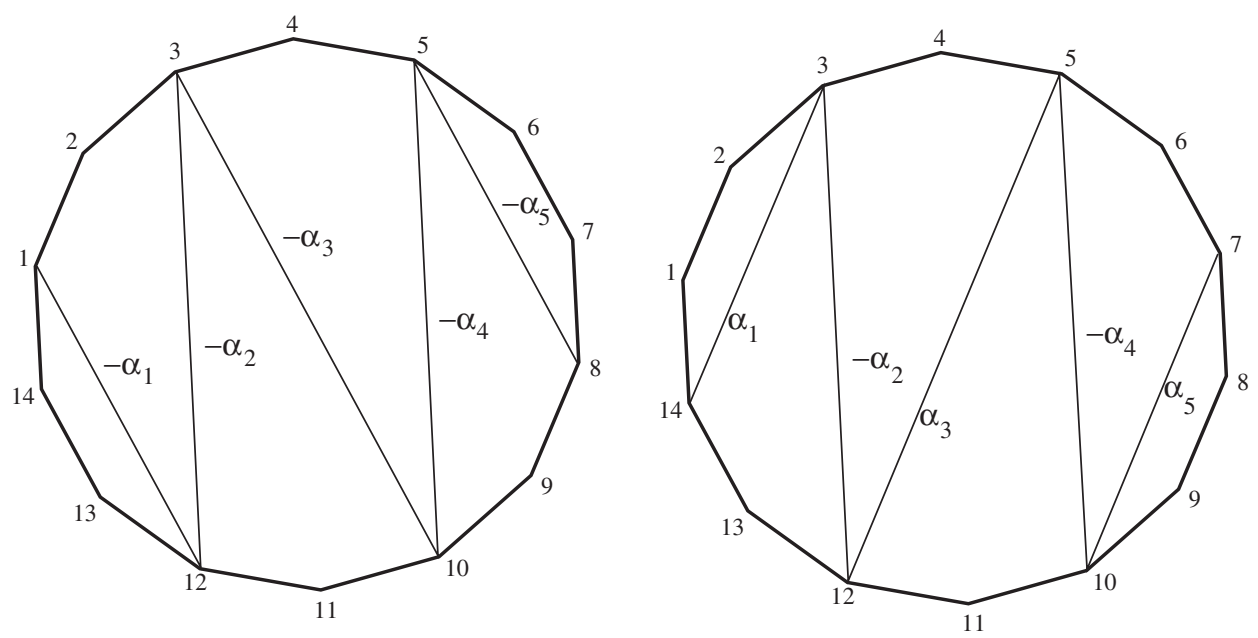

Figure 3. $m$-snake and opposite $m$-snake for $n=6, m=2$

Let $I=I^{+} \cup I^{-}$be a decomposition of the vertices $I$ of $G$ so that there are no arrows between vertices in $I^{+}$or between vertices in $I^{-}$; such a decomposition exists because $G$ is bipartite. For type $A_{n-1}$, we take $I^{+}$to be the even-numbered vertices and $I^{-}$to be the odd-numbered vertices.

Let $R_{m}: \Phi_{\geq-1}^{m} \rightarrow \Phi_{\geq-1}^{m}$ be the bijection introduced by Fomin-Reading [FR, 2.3]. This is defined using the involutions [FZ2] $\tau_{ \pm}: \Phi_{\geq-1} \rightarrow \Phi_{\geq-1}$ given by

$$
\tau_{\varepsilon}(\beta)=\left\{\begin{array}{cc}
\alpha & \text { if } \beta=-\alpha_{i}, \text { for } i \in I^{-\varepsilon}, \\
\left(\prod_{i \in I^{\varepsilon}} s_{i}\right)(\beta) & \text { otherwise. }
\end{array}\right.
$$

Then, for $\beta^{k} \in \Phi_{\geq-1}^{m}$, we have

$$
R_{m}\left(\beta^{k}\right)=\left\{\begin{array}{cc}
\beta^{k+1} & \text { if } \alpha \in \Phi^{+} \text {and } k<m, \\
\left(\left(\tau_{-} \tau_{+}\right)(\beta)\right)^{1} & \text { otherwise. }
\end{array}\right.
$$

Lemma 4.1 (Fomin-Reading). For all $\beta^{k} \in \Phi_{\geq-1}^{m}$, we have: $D\left(R_{m}\left(\beta^{k}\right)\right)=$ $R_{m} D\left(\beta^{k}\right)$.

Proof. See the discussion in [FR, 4.1].

4.2. Indecomposable objects in the $m$-cluster category and $m$-diagonals. Let $Q_{\text {alt }}$ denote the orientation of $G$ obtained by orienting every arrow to go from a vertex in $I^{+}$to a vertex in $I^{-}$, so that the vertices in $I^{+}$are sources and the vertices in $I^{-}$are sinks.

For a positive root $\alpha$, let $V(\alpha)$ denote the corresponding $k Q_{a l t}$-module, regarded as an indecomposable object in $D^{b}\left(k Q_{a l t}\right)$. Then it is clear from the definition that 
the indecomposable objects in $\mathcal{C}_{G}^{m}$ are the objects $S^{k-1} V(\alpha)$ for $k=1,2, \ldots, m$, $\alpha \in \Phi^{+}$, and $S^{-1} I_{i}$ for $I_{i}$ an indecomposable injective $k Q_{a l t}$-module corresponding to the vertex $i \in I$ (all regarded as objects in the $m$-cluster category). Following Thomas [Tho] or Zhu [Zhu], we define $V\left(\alpha^{k}\right)$ to be $S^{k-1} V(\alpha)$ for $k=1,2, \ldots, m$, $\alpha \in \Phi^{+}$, and $V\left(-\alpha_{i}\right)=S^{-1} I_{i}$ for $i \in I$.

We have:

Lemma 4.2 (Thomas, Zhu). For all $\beta^{k} \in \Phi_{\geq-1}^{m}, V\left(R_{m} \beta^{k}\right) \cong S V\left(\beta^{k}\right)$, where $S$ denotes the autoequivalence of $\mathcal{C}_{G}^{m}$ induced by the shift on $D^{b}(k Q)$.

Proof. See [Tho, Lemma 2] or [Zhu, 3.8].

\section{An ISOMORPHISM OF STABLE TRANSLATION QUIVERS}

From the previous two sections, we see that in type $A_{n-1}$ we have a bijection $D$ from $\Phi_{>-1}^{m}$ to the set of $m$-diagonals of $\Pi$ and a bijection $V$ from $\Phi_{>-1}^{m}$ to the objects of ind $\mathcal{C}_{A_{n-1}}^{m}$ up to isomorphism, i.e. to the vertices of the Auslander-Reiten quiver of $\mathcal{C}_{A_{n-1}}^{m}$. Composing the inverse of $D$ with $V$ we obtain a bijection $\psi$ from the set of $m$-diagonals of $\Pi$ to ind $\mathcal{C}_{A_{n-1}}^{m}$.

Lemma 5.1. For every $m$-diagonal $D$ of $\Pi$, we have that

$$
\psi\left(R_{m}(D)\right) \cong S \psi(D),
$$

and therefore that

$$
\psi\left(\tau_{m}(D)\right) \cong \tau(\psi(D)) .
$$

Proof. The first statement follows immediately from Lemmas 4.1 and 4.2. We can deduce from this that $\psi\left(\tau_{m}(D)\right)=\psi\left(R_{m}^{m}(D)\right)=S^{m} \psi(D)$ and thus obtain the second statement, since $S^{m}$ coincides with $\tau$ on every indecomposable object of $\mathcal{C}_{A_{n-1}}^{m}$ by the definition of this category.

It remains to show that $\psi$ and $\psi^{-1}$ are morphisms of quivers.

Lemma 5.2. - For $1 \leq i \leq \frac{n-1}{2}$, there is an arrow in $\Gamma_{A_{n-1}}^{m}$ from $D\left(-\alpha_{2 i-1}\right)$ to $D\left(-\alpha_{2 i}\right)$.

- For $1 \leq i \leq \frac{n-1}{2}$, there is an arrow in $\Gamma_{A_{n-1}}^{m}$ from $D\left(-\alpha_{2 i+1}\right)$ to $D\left(-\alpha_{2 i}\right)$.

- For $1 \leq i \leq \frac{n}{2}$, there is an arrow in $\Gamma_{A_{n-1}}^{m}$ from $D\left(-\alpha_{2 i}\right)$ to $D\left(\alpha_{2 i-1}^{1}\right)$.

- For $1 \leq i \leq \frac{n-2}{2}$, there is an arrow in $\Gamma_{A_{n-1}}^{m}$ from $D\left(-\alpha_{2 i}\right)$ to $D\left(\alpha_{2 i+1}^{1}\right)$.

These are the only arrows amongst the diagonals $D\left(-\alpha_{i}\right)$ and $D\left(\alpha_{j}^{1}\right)$, for $1 \leq$ $i, j \leq n-1$, with $j$ odd, in $\Gamma_{A_{n-1}}^{m}$.

Proof. We first note that, for $1 \leq i \leq \frac{n-1}{2}$, the diagonals corresponding to the negative simple roots $-\alpha_{2 i-1}$ and $-\alpha_{2 i}$, together with an arc of the boundary containing vertices $(i-1) m+1, \ldots, i m+1$, bound an $m+2$-gon. The other vertex is numbered $(n-i) m+2$. Furthermore, $D\left(-\alpha_{2 i-1}\right)$ can be rotated clockwise about the common endpoint $(n-i) m+2$ to $D\left(-\alpha_{2 i}\right)$, so there is an arrow in $\Gamma_{A_{n-1}}^{m}$ from $D\left(-\alpha_{2 i-1}\right)$ to $D\left(-\alpha_{2 i}\right)$.

Similarly, for $1 \leq i \leq \frac{n-2}{2}$, the diagonals corresponding to the negative simple roots $-\alpha_{2 i}$ and $-\alpha_{2 i+1}$, together with an arc of the boundary containing vertices $(n-i-1) m+2, \ldots,(n-i) m+2$, bound an $m+2$-gon (with the other vertex being numbered $i m+1)$, and $D\left(-\alpha_{2 i+1}\right)$ can be rotated clockwise about the common 
endpoint $i m+1$ to $D\left(-\alpha_{2 i}\right)$, so there is an arrow in $\Gamma_{A_{n-1}}^{m}$ from $D\left(-\alpha_{2 i+1}\right)$ to $D\left(-\alpha_{2 i}\right)$.

We have observed that, for $1 \leq i \leq \frac{n}{2}$, the coloured root $\alpha_{2 i-1}^{1}$ corresponds to the diagonal $(i m+1,(n+1-i) m+2)$. Consideration of the $m+2$-gon with vertices $(n-i) m+2, \ldots,(n+1-i) m+2$ and $i m+1$ shows that there is an arrow from $D\left(-\alpha_{2 i}\right)$ to $D\left(\alpha_{2 i-1}^{1}\right)$. For $1 \leq i \leq \frac{n-2}{2}$, consideration of the $m+2$-gon with vertices $i m+1, \ldots(i+1) m+1$ and $(n-i) m+2$ shows that there is an arrow from $D\left(-\alpha_{2 i}\right)$ to $D\left(\alpha_{2 i+1}^{1}\right)$.

The statement that these are the only arrows amongst the diagonals considered is clear.

The following follows from the well-known structure of the Auslander-Reiten quiver of $D^{b}(k Q)$.

Lemma 5.3. - For $1 \leq i \leq \frac{n-1}{2}$, there is an arrow in the Auslander-Reiten quiver of $\mathcal{C}_{A_{n-1}}^{m}$ from $I_{2 i-1}[-1]$ to $I_{2 i}[-1]$.

- For $1 \leq i \leq \frac{n-2}{2}$, there $i s$ an arrow from $I_{2 i+1}[-1]$ to $I_{2 i}[-1]$.

- For $1 \leq i \leq \frac{n}{2}$, there is an arrow from $I_{2 i}[-1]$ to $P_{2 i-1}$.

- For $1 \leq i \leq \frac{n-2}{2}$ there is an arrow from $I_{2 i}[-1]$ to $P_{2 i+1}$.

These are the only arrows amongst the vertices $I_{i}[-1]$ and $P_{j}$ for $1 \leq i, j \leq n-1$, with $j$ odd, in the Auslander-Reiten quiver of $\mathcal{C}_{A_{n-1}}^{m}$.

Proposition 5.4. The map $\psi$ from m-diagonals in $\Pi$ to indecomposable objects in $\mathcal{C}_{A_{n-1}}^{m}$ is an isomorphism of quivers.

Proof. Suppose that $D, E$ are $m$-diagonals in $\Pi$ and that there is an arrow from $D$ to $E$. Write $D=D\left(\beta^{k}\right)$ and $E=D\left(\gamma^{l}\right)$ for coloured roots $\beta^{k}$ and $\gamma^{l}$. Then $V:=\psi(D)=V\left(\beta^{k}\right)$ and $W:=\psi(E)=V\left(\gamma^{l}\right)$ are corresponding vertices in the Auslander-Reiten quiver of $\mathcal{C}_{A_{n-1}}^{m}$. Since there is an arrow from $D$ to $E$, there is an $m+2$-gon bounded by $D$ and $E$ and an arc of the boundary of $\Pi$.

Since $D$ is an $m$-diagonal, on the side of $D$ not in the $m+2$-gon, there is a $d m+2$ gon bounded by $D$ and an arc of the boundary of $\Pi$ for some $d \geq 1$. Similarly, since $E$ is an $m$-diagonal, on the side of $E$ not in the $m+2$-gon, there is an $\mathrm{em}+2$-gon bounded by $D$ and an arc of the boundary of $\Pi$, for some $e \geq 1$. It is clear that each of these polygons can be dissected by an $m$-snake such that, together with $D$ and $E$, we obtain a 'zig-zag' dissection $\chi$ of $\Pi$. Let $v$ be one of its endpoints. The other endpoint of the diagonal containing $v$ must be $v-m-1$ or $v+m+1$ (modulo $n m+2)$.

In the first case, we have that for some $t \in \mathbb{Z}, R_{m}^{t}(v)=1$ and $R_{m}^{t}$ applied to $\chi$ is the $m$-snake. In the second case, we have that, for some $t \in \mathbb{Z}, R_{m}^{t}(v)=n m+2$ and $R_{m}^{t}$ applied to $\chi$ is the opposite $m$-snake. It follows from Lemma 5.3 that there is an arrow from $R_{m}^{t}(V)$ to $R_{m}^{t}(W)$ in the Auslander-Reiten quiver of $\mathcal{C}_{A_{n-1}}^{m}$, and hence from $V$ to $W$.

Conversely, suppose that $V, W$ are vertices of the Auslander-Reiten quiver of $\mathcal{C}_{A_{n-1}}^{m}$ and that there is an arrow from $V$ to $W$. We can write $V=V\left(\beta^{k}\right)$ and $W=V\left(\gamma^{l}\right)$ for coloured roots $\beta^{k}$ and $\gamma^{l}$. Let $D:=\psi^{-1}(V)=D\left(\beta^{k}\right)$ and let $E:=\psi^{-1}(W)=D\left(\gamma^{l}\right)$. It is clear that $\tau^{u}(V) \cong I_{i}[-1]$ for some $i$ and some $u$. By Lemma 5.3, we must have that either $\tau^{u}(W) \cong I_{i \pm 1}[-1]$ or $\tau^{u}(W) \cong P_{i \pm 1}$. In the latter case we must have that $i$ is even. Note that $S^{u m}(V) \cong \tau^{u}(V)$ and 
$S^{u m}(W) \cong \tau^{u}(W)$. It follows from Lemmas 5.1 and 5.2 that there is an arrow from $R_{m}^{u m}(D)$ to $R_{m}^{u m}(E)$ in $\Gamma_{A_{n-1}}^{m}$, and thus from $D$ to $E$.

It follows that $\psi$ is an isomorphism of quivers.

Proposition 5.5. There is an isomorphism $\psi$ of translation quivers between the stable translation quiver $\Gamma_{A_{n-1}}^{m}$ of $m$-diagonals and the Auslander-Reiten quiver of the $m$-cluster category $\mathcal{C}_{A_{n-1}}^{m}$.

Proof. This now follows immediately from Proposition 5.4 and Lemma 5.1.

We therefore have our main result.

Theorem 5.6. The $m$-cluster category $\mathcal{C}_{A_{n-1}}^{m}$ is equivalent to the additive category generated by the mesh category of the stable translation quiver $\Gamma_{A_{n-1}}^{m}$ of $m$ diagonals.

We remark that a connection between the $m$-cluster category and the $m$-diagonals has been given in [Tho]. In particular, Thomas gives an interpretation of Ext-groups in the $m$-cluster category in terms of crossings of diagonals. However, Thomas does not give a construction of the $m$-cluster category using diagonals.

\section{THE $m$-TH POWER OF A TRANSLATION QUIVER}

In this section we define a new category in a natural way in which the $m$-cluster category $\mathcal{C}_{A_{n-1}}^{m}$ will appear as a full subcategory. We start with a translation quiver $\Gamma$ and define its $m$-th power.

Let $\Gamma$ be a translation quiver with translation $\tau$.

Let $\Gamma^{m}$ be the quiver whose objects are the same as the objects of $\Gamma$ and whose arrows are the sectional paths of length $m$. A path $\left(x=x_{0} \rightarrow x_{1} \rightarrow \cdots \rightarrow x_{m-1} \rightarrow\right.$ $x_{m}=y$ ) in $\Gamma$ is said to be sectional if $\tau x_{i+1} \neq x_{i-1}$ for $i=1, \ldots, m-1$ (for which $\tau x_{i+1}$ is defined) (cf. [Rin]). Let $\tau^{m}$ be the $m$-th power of the translation, i.e. $\tau^{m}=\tau \circ \tau \circ \cdots \circ \tau$ ( $m$ times). Note that the domain of the definition of $\tau^{m}$ is a subset of the domain of the definition $\Gamma_{0}^{\prime}$ of $\tau$.

Recall that a translation quiver is said to be hereditary (see [Rin]) if:

- for any non-projective vertex $z$, there is an arrow from some vertex $z^{\prime}$ to $z$;

- there is no (oriented) cyclic path of length at least one containing projective vertices, and

- if $y$ is a projective vertex and there is an arrow $x \rightarrow y$, then $x$ is projective.

The last condition is what we need to ensure that $\left(\Gamma^{m}, \tau^{m}\right)$ is again a translation quiver:

Theorem 6.1. Let $(\Gamma, \tau)$ be a translation quiver such that if $y$ is a projective vertex and there is an arrow $x \rightarrow y$, then $x$ is projective. Then $\left(\Gamma^{m}, \tau^{m}\right)$ is a translation quiver.

Proof. We prove the following statement by induction on $m$ :

Suppose that there is a sectional path

$$
x=x_{0} \rightarrow x_{1} \rightarrow \cdots \rightarrow x_{m}=y
$$

in $\Gamma$ and $\tau^{m} y$ is defined. Then $\tau^{i} x_{i}$ is defined for $i=0,1, \ldots, m$ and there is a sectional path

$$
\tau^{m} y=\tau^{m} x_{m} \rightarrow \tau^{m-1} x_{m-1} \rightarrow \cdots \rightarrow \tau x_{1} \rightarrow x=x_{0}
$$


in $\Gamma$. Furthermore, if the multiplicities of arrows between consecutive vertices in the first path are $k_{1}, k_{2}, \ldots, k_{m}$, the multiplicities of arrows between consecutive vertices in the second path are $k_{m}, k_{m-1}, \ldots, k_{1}$.

This is clearly true for $m=1$, since $\Gamma$ is a translation quiver. Suppose it is true for $m-1$, and that

$$
x=x_{0} \rightarrow x_{1} \rightarrow \cdots \rightarrow x_{m}=y
$$

is a sectional path in $\Gamma$. Since $\tau^{m-1} x_{m}$ is defined, we can apply induction to the sectional path,

$$
x_{1} \rightarrow x_{2} \rightarrow \cdots \rightarrow x_{m},
$$

to obtain that $\tau^{i-1} x_{i}$ is defined for $i=1,2, \ldots, m$ and that there is a sectional path

$$
\tau^{m-1} x_{m} \rightarrow \tau^{m-2} x_{m-1} \rightarrow \cdots x_{1}
$$

in $\Gamma$, with multiplicities $k_{2}, k_{3}, \ldots, k_{m}$. As $\tau^{m} x_{m}$ is defined, $\tau^{m-1} x_{m}$ is not projective, and it follows that $\tau^{i-1} x_{i}$ is not projective for $i=1,2, \ldots, m$ by our assumption. Therefore $\tau^{i} x_{i}$ is defined for $i=1,2, \ldots, m$. For $i=2,3, \ldots, m$, there are $k_{i}$ arrows from $\tau^{i-1} x_{i}$ to $\tau^{i-2} x_{i-1}$. Therefore there are $k_{i}$ arrows from $\tau^{i-1} x_{i-1}$ to $\tau^{i-1} x_{i}$. Thus there are $k_{i}$ arrows from $\tau^{i} x_{i}$ to $\tau^{i-1} x_{i-1}$. As there are $k_{1}$ arrows from $x_{0}$ to $x_{1}$, there are $k_{1}$ arrows from $\tau x_{1}$ to $x_{0}$. If $\tau\left(\tau^{i} x_{i}\right)=\tau^{i+2} x_{i+2}$ for some $i$, then $x_{i}=\tau x_{i+2}$, contradicting the fact that $x_{0} \rightarrow x_{1} \rightarrow \cdots \rightarrow y$ is sectional. It follows that

$$
\tau^{m} x_{m} \rightarrow \tau^{m-1} x_{m-1} \rightarrow \cdots \rightarrow x_{0}=x
$$

is a sectional path with multiplicities of arrows $k_{1}, k_{2}, \ldots, k_{m}$ as required.

It follows that the number of sectional paths with sequence of vertices $x_{0}, x_{1}, \ldots$, $x_{m}$ is less than or equal to the number of sectional paths with a sequence of vertices $\tau^{m} y=\tau^{m} x_{m}, \tau^{m-1} x_{m-1}, \ldots, \tau x_{1}, x_{0}=x$.

Suppose that

$$
x=x_{0}^{\prime} \rightarrow x_{1}^{\prime} \rightarrow \cdots \rightarrow x_{m}^{\prime}=y
$$

is a sectional path from $x$ to $y$ with a different sequence of vertices. Then $x_{i} \neq x_{i}^{\prime}$ for some $i, 0<i<m$. It follows that $\tau^{i} x_{i} \neq \tau^{i} x_{i}^{\prime}$ and thus that the sectional path from $\tau^{m} y$ to $x$ provided by the above argument is also on a different sequence of vertices. Thus, applying the above argument to every sectional path of length $m$ from $x$ to $y$, we obtain an injection from the set of sectional paths of length $m$ from $x$ to $y$ to the set of sectional paths of length $m$ from $\tau^{m} y$ to $x$.

A similar argument shows that whenever there is a sectional path

$$
\tau^{m} y=y_{0} \rightarrow y_{1} \rightarrow \cdots \rightarrow y_{m}=x
$$

in $\Gamma$ with multiplicities $l_{1}, l_{2}, \ldots, l_{m}$, then $\tau^{i-m} y_{i}$ is defined for all $i$ and there is a sectional path

$$
x \rightarrow \tau^{-1} y_{m-1} \rightarrow \cdots \rightarrow \tau^{m-1} y_{1} \rightarrow \tau^{m} y=y_{0}
$$

in $\Gamma$ with multiplicities $l_{m}, l_{m-1}, \ldots, l_{1}$, and as above we obtain an injection from the set of sectional paths of length $m$ from $\tau^{m} y$ to $x$ to the set of sectional paths of length $m$ from $x$ to $y$.

Since $\Gamma$ is locally finite, the number of sectional paths of fixed length between two vertices is finite. It follows that the number of sectional paths of length $m$ from $x$ to $y$ is the same as the number of sectional paths of length $m$ from $\tau^{m} y$ to $x$. Hence $\left(\Gamma^{m}, \tau^{m}\right)$ is a translation quiver. 
We remark that the square of the translation quiver below, which does not satisfy the additional assumption of the theorem, is not a translation quiver:

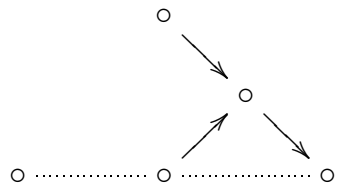

Corollary 6.2. (1) Let $(\Gamma, \tau)$ be a hereditary translation quiver. Then $\left(\Gamma^{m}, \tau^{m}\right)$ is a translation quiver.

(2) Let $(\Gamma, \tau)$ be a stable translation quiver. Then $\left(\Gamma^{m}, \tau^{m}\right)$ is a stable translation quiver.

Proof. Part (1) is immediate from Theorem 6.1 and the definition of a hereditary translation quiver. For $(2)$, note that if $(\Gamma, \tau)$ is stable, no vertex is projective, so $\left(\Gamma^{m}, \tau^{m}\right)$ is a translation quiver by Theorem 6.1. Since $\tau$ is defined on all vertices of $\Gamma$, so is $\tau^{m}$.

We remark that the $m$-th power of a hereditary translation quiver need not be hereditary: there can be non-projective vertices $z$ without any vertex $z^{\prime}$ such that $z^{\prime} \rightarrow z$. For example, consider the hereditary translation quiver below. It is clear that its square in the above sense has no arrows, but does have non-projective vertices.

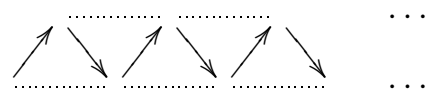

However, we do have the following:

Proposition 6.3. Let $(\Gamma, \tau)$ be a translation quiver such that for any arrow $x \rightarrow y$ in $\Gamma, x$ is projective whenever $y$ is projective. Then the translation quiver $\left(\Gamma^{m}, \tau^{m}\right)$ has the same property.

Proof. We know by Theorem 6.1 that $\left(\Gamma^{m}, \tau^{m}\right)$ is a translation quiver. Suppose that

$$
x_{0}=x \rightarrow x_{1} \rightarrow \cdots \rightarrow x_{m}=y
$$

is a sectional path in $\Gamma$ and that $\tau^{m} x$ is defined, i.e. $x$ is not projective in $\left(\Gamma^{m}, \tau^{m}\right)$. Then $\tau x$ is defined, so $x$ is not projective in $(\Gamma, \tau)$. Hence $x_{1}, x_{2}, \ldots, x_{m}$ are not projective in $(\Gamma, \tau)$. Since there are arrows $x_{i-1} \rightarrow x_{i}$ for $i=1,2, \ldots, m$, there are arrows $\tau x_{i} \rightarrow x_{i-1}$ and therefore arrows $\tau x_{i-1} \rightarrow \tau x_{i}$ for $i=1,2, \ldots, m$. Repeating this argument we see that $\tau^{m} x_{i}$ is defined for all $i$. In particular, $\tau^{m} x_{m}$ is defined, so $y=x_{m}$ is not projective in $\left(\Gamma^{m}, \tau^{m}\right)$, and we are done.

\section{The $m$-Cluster CATEgORY IN TERMS OF $m$-TH POWERS}

We consider the construction of Section 6 in the case where $\Gamma$ is the quiver given by the diagonals of an $N$-gon $\Pi$, i.e. $\Gamma=\Gamma_{A_{N-3}}^{1}$ as in Section 2 . Here, we fix $m=1$, i.e. the vertices of the quiver are the usual diagonals of $\Pi$, and there is an arrow from $D$ to $D^{\prime}$ if $D, D^{\prime}$ have a common endpoint $i$ so that $D, D^{\prime}$, together with the arc from $j$ to $j^{\prime}$ between the other endpoints, form a triangle and $D$ is rotated to $D^{\prime}$ by a clockwise rotation about $i$. We will call this rotation $\rho_{i}$. Furthermore, we have introduced an automorphism $\tau_{1}$ of $\Gamma$ : $\tau_{1}$ sends $D$ to $D^{\prime}$ if $D$ can be rotated to 
$D^{\prime}$ by an anticlockwise rotation about the centre of the polygon through $\frac{2 \pi}{N}$. Then $\Gamma=\Gamma_{A_{N-3}}^{1}$ is a stable translation quiver (cf. Proposition 2.2).

The geometric interpretation of a sectional path of length $m$ from $D$ to $D^{\prime}$ is given by the map $\rho_{i}^{m}: \rho_{i}^{m}$ sends the diagonal $D$ to $D^{\prime}$ if $D, D^{\prime}$ have a common endpoint $i$ and, together with the arc between the other endpoints $j, j^{\prime}$, form an $m+2$-gon, and if $D$ can be rotated to $D^{\prime}$ with a clockwise rotation about the common endpoint.

Furthermore, the $m$-th power $\tau_{1}^{m}$ of the translation $\tau_{1}$ corresponds to a anticlockwise rotation through $\frac{2 m \pi}{N}$ about the centre of the polygon. From that one obtains:

Proposition 7.1. 1) The quiver $\left(\Gamma_{A_{N-3}}^{1}\right)^{m}$ contains a translation quiver of $m$ diagonals if and only if $N=n m+2$ for some $n$.

2) $\Gamma_{A_{n-1}}^{m}$ is a connected component of $\left(\Gamma_{A_{n m-1}}^{1}\right)^{m}$.

Proof. 1) Note that if $N \neq n m+2$ (for some $n$ ), then $\Gamma_{A_{N-3}}^{m}$ contains no $m$ diagonals.

So assume that $N=n m+2$ for some $n$. Let $\Gamma:=\Gamma_{A_{N-3}}^{1}=\Gamma_{A_{n m-1}}^{1}$. We have to show that $\Gamma^{m}$ contains $Q:=\Gamma_{A_{n-1}}^{m}$. Recall that the vertices of the quiver $\Gamma^{m}$ are the diagonals of an $n m+2$-gon and that $Q$ is the quiver whose vertices are the $m$-diagonals of an $n m+2$-gon. So the vertices of $Q$ are vertices of $\Gamma^{m}$.

We claim that the arrows between those vertices are the same for $Q$ and for $\Gamma^{m}$. In other words, we claim that there is a sectional path of length $m$ between $D$ and $D^{\prime}$ if and only if $D$ can be rotated clockwise to $D^{\prime}$ about a common endpoint and $D$ and $D^{\prime}$, together with an arc joining the other endpoints, bound an $(m+2)$-gon.

Let $D \rightarrow D^{\prime}$ be an arrow in $\Gamma^{m}$, where $D$ is the diagonal $(i, j)$ from $i$ to $j$. Without loss of generality, let $i<j$. The arrow $D \rightarrow D^{\prime}$ in $\Gamma^{m}$ corresponds to a sectional path of length $m$ in $\Gamma, D \rightarrow D_{1} \rightarrow \cdots \rightarrow D_{m-1} \rightarrow D_{m}=D^{\prime}$. We describe such sectional paths. The first arrow is either $D=(i, j) \rightarrow(i, j+1)$ or $(i, j) \rightarrow(i-1, j)$, i.e. $D_{1}=(i, j+1)$ or $D_{1}=(i-1, j)($ vertices taken $\bmod N)$. In the first case, one then gets an arrow $D_{1}=(i, j+1) \rightarrow(i+1, j+1)$ or $D_{1} \rightarrow(i, j+2)$. Now $\tau(i+1, j+1)=(i, j)$, and since the path is sectional, we get that $D_{2}$ can only be the diagonal $(i, j+2)$.

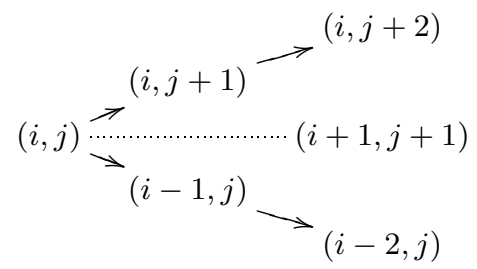

Repeatedly using the above argument, we see that the sectional path has to be of the form

$$
D=D_{0}=(i, j) \rightarrow(i, j+1) \rightarrow(i, j+2) \rightarrow \cdots \rightarrow(i, j+m)=D_{m}=D^{\prime}
$$

where all vertices are taken $\bmod N$.

Similarly, if $D_{1}=(i-1, j)$, then $D_{2}=(i-2, j)$ and so on, and $D_{m}=(i-m, j)$ $(\bmod N)$.

In particular, in the first case, the arrow $D \rightarrow D^{\prime}$ corresponds to a rotation $\rho_{i}^{m}$ about the common endpoint $i$ of $D, D^{\prime}$. In the second case, the arrow $D \rightarrow D^{\prime}$ 
corresponds to $\rho_{j}^{m}$. In each case $D, D^{\prime}$ and an arc between them bound an $(m+2)$ gon, so there is an arrow from $D$ to $D^{\prime}$ in $Q$.

Since it is clear that every arrow in $Q$ arises in this way, we see that the arrows between the vertices of $Q$ and of the corresponding subquiver of $\Gamma^{m}$ are the same.

2) We know by Proposition 2.3 that $Q=\Gamma_{A_{n-1}}^{m}$ is a connected stable translation quiver. If there is an arrow $D \rightarrow D^{\prime}$ in $\Gamma^{m}$ where $D$ is an $m$-diagonal, then $D^{\prime}$ is an $m$-diagonal. Similarly, $\tau_{1}^{m}(D)$ is also an $m$-diagonal.

Theorem 7.2. The $m$-cluster category $\mathcal{C}_{A_{n-1}}^{m}$ is a full subcategory of the additive category generated by the mesh category of $\left(\Gamma_{A_{n m-1}}^{1}\right)^{m}$.

Proof. This is a consequence of Proposition 7.1 and Theorem 5.6

Remark 7.3. Even if $\Gamma$ is a connected quiver, $\Gamma^{m}$ need not be connected. As an example we consider the quiver $\Gamma=\Gamma_{A_{5}}^{1}$ and its second power $\left(\Gamma_{A_{5}}^{1}\right)^{2}$ pictured in Figures 4 and 5. The connected components of $\left(\Gamma_{A_{5}}^{1}\right)^{2}$ are $\Gamma_{A_{2}}^{2}$ and two copies of a translation quiver whose mesh category is equivalent to ind $D^{b}\left(A_{3}\right) /[1]$ (where $D^{b}\left(A_{3}\right)$ denotes the derived category of a Dynkin quiver of type $\left.A_{3}\right)$. We thus obtain a geometric construction of a quotient of $D^{b}\left(A_{3}\right)$ which is not an $m$-cluster category.

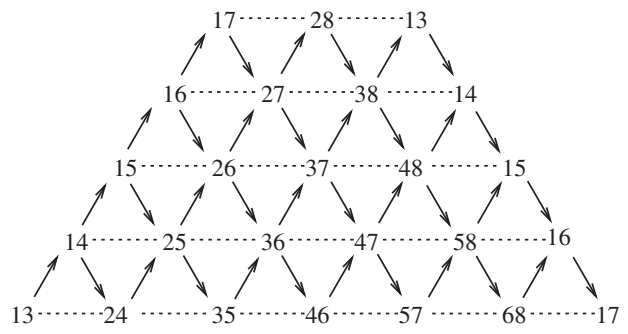

Figure 4. The quiver $\Gamma_{A_{5}}^{1}$
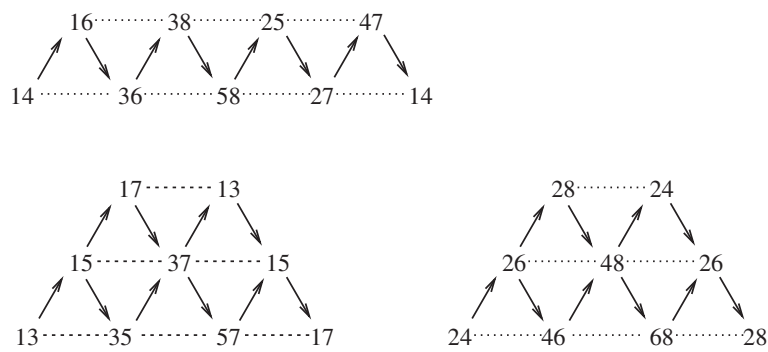

FiguRE 5. The three components of $\left(\Gamma_{A_{5}}^{1}\right)^{2}$

\section{REFERENCES}

[BMRRT] A. B. Buan, R. J. Marsh, M. Reineke, I. Reiten and G. Todorov. Tilting theory and cluster combinatorics. Preprint arxiv:math.RT/0402054, February 2004, to appear in Adv. Math. MR2249625 (2007f:16033) 
[CCS1] P. Caldero, F. Chapoton and R. Schiffler. Quivers with relations arising from clusters $\left(A_{n}\right.$ case). Trans. Amer. Math. Soc. 358 (2006), no. 3, 1347-1364. MR2187656 (2007a:16025)

[CCS2] P. Caldero, F. Chapoton and R. Schiffler. Quivers with relations and cluster tilted algebras. J. Alg. Rep. Theory 9 (2006), no. 4, 359-376. MR2250652 (2007f:16036)

[FR] S. Fomin and N. Reading. Generalized cluster complexes and Coxeter combinatorics. International Mathematics Research Notices 2005:44, 2709-2757. MR2181310 (2006g:05230)

[FZ1] S. Fomin and A. Zelevinsky. Cluster algebras I: Foundations. J. Amer. Math. Soc. 15 (2002), no. 2, 497-529. MR1887642 (2003f:16050)

[FZ2] S. Fomin and A. Zelevinsky. Y-systems and generalized associahedra. Annals of Mathematics. 158 (2003), no. 3, 977-1018. MR2031858 (2004m:17010)

[Hap] D. Happel. Triangulated categories in the representation theory of finite dimensional algebras. LMS Lecture Note Series 119, Cambridge University Press, 1988. MR935124 (89e:16035)

[Kel] B. Keller. On triangulated orbit categories. Documenta Math. 10 (2005), 551-581. MR2184464 (2007c:18006)

[KR] B. Keller and I. Reiten. Cluster-tilted algebras are Gorenstein and stably Calabi-Yau. Preprint arxiv:math.RT/0512471, December 2005. MR2313531

[PS] J. Przytycki and A. Sikora. Polygon dissections and Euler, Fuss, Kirkman and Cayley numbers. J. Combin. Theory Series A 92 (2000), 68-76. MR1783940 (2001g:05005)

[Rie] C. Riedtmann. Algebren, Darstellungsköcher, Überlagerungen und zurück. Comment. Math. Helv. 55 (1980), no. 2, 199-224. MR576602 (82k:16039)

[Rin] C.M. Ringel. Tame Algebras and Integral Quadratic Forms. Lecture Notes in Mathematics 1099 (1984), Springer, Berlin. MR774589 (87f:16027)

[Tho] H. Thomas. Defining an m-cluster category. Preprint arxiv:math.RT/0607173v1, 2005.

[Tza] E. Tzanaki. Polygon dissections and some generalizations of cluster complexes. Preprint arxiv:math.CO/0501100, January 2005. MR2244140 (2007c:05201)

[Wra] A. Wralsen. Ph.D. Thesis, in preparation.

[Zhu] B. Zhu. Generalized cluster complexes via quiver representations. Preprint arxiv:math.RT/0607155, July 2006.

Department of Mathematics, University of Leicester, University Road, Leicester LE1 7RH, ENGLAND

E-mail address: k.baur@mcs.le.ac.uk

Current address: Department of Mathematics, ETH Zürich, Rämistrasse 101, CH-8092 Zürich, Switzerland

Department of Pure Mathematics, University of Leeds, Leeds LS2 9JT, England 